\title{
CR YAMABE CONSTANT, CR YAMABE FLOW AND ITS SOLITON
}

\author{
PAK TUNG HO AND KUNBO WANG
}

\begin{abstract}
On a compact strictly pseudoconvex CR manifold $(M, \theta)$, we consider the CR Yamabe constant of its infinite conformal covering. By using the maximum principles, we then prove a uniqueness theorem for the CR Yamabe flow on a complete noncompact CR manifold. Finally we obtain some properties of the CR Yamabe soliton on complete noncompact CR manifolds.
\end{abstract}

\section{INTRODUCTON}

Let $(M, \theta)$ be a compact pseudoconvex manifold of real dimension $2 n+1$ with the contact form $\theta$. Given $0<u \in C^{\infty}(M)$, we can define the energy by

$$
E_{(M, \theta)}(u)=\int_{M}\left(\left(2+\frac{2}{n}\right)\left|\nabla_{\theta} u\right|^{2}+R_{\theta} u^{2}\right) d V_{\theta} /\left(\int_{M} u^{2+\frac{2}{n}} d V_{\theta}\right)^{\frac{n}{n+1}} .
$$

The CR Yamabe constant of $(M, \theta)$ is then defined as

$$
Y(M, \theta)=\inf \left\{E_{(M, \theta)}(u) \mid 0<u \in C^{\infty}(M)\right\} .
$$

It follows from the definition that the CR Yamabe constant depends only on the conformal class of $\theta$. The CR Yamabe problem is to find a contact form in the conformal class such that its Tanaka-Webster scalar curvature is constant. If we write $\tilde{\theta}=u^{\frac{2}{n}} \theta$ for some $0<u \in C^{\infty}(M)$, then their Tanaka-Webster scalar curvatures are related by

$$
-\left(2+\frac{2}{n}\right) \Delta_{\theta} u+R_{\theta} u=R_{\tilde{\theta}} u^{1+\frac{2}{n}} .
$$

Therefore, the CR Yamabe problem is to find a positive smooth function $u$ in $M$ satisfying (1.2) with $R_{\tilde{\theta}}$ being constant. The following theorem was proved by Jerison and Lee: (See Theorem 3.4 in [10])

Theorem 1.1 (Jerison-Lee). Given a compact $C R$ manifold $(M, \theta)$, we have (i) $Y(M, \theta) \leq Y\left(S^{2 n+1}, \theta_{S^{2 n+1}}\right)$ where $Y\left(S^{2 n+1}, \theta_{S^{2 n+1}}\right)$ is the CR Yamabe constant of the $C R$ sphere $\left(S^{2 n+1}, \theta_{S^{2 n+1}}\right)$; and

(ii) if $Y(M, \theta)<Y\left(S^{2 n+1}, \theta_{S^{2 n+1}}\right)$ then the infimum in (1.1) is attained by a positive $C^{\infty}$ solution to (1.2). Thus, the contact form $\tilde{\theta}=u^{\frac{2}{n} \theta}$ has constant TanakaWebster scalar curvature.

In view of Theorem 1.1, it is important to estimate the CR Yamabe constant $Y(M, \theta)$. In section 3, we consider the CR Yamabe constant of conformal covering of $(M, \theta)$.

Date: 28th July, 2019.

2010 Mathematics Subject Classification. 32V05,32V20,53C44.

Key words and phrases. CR Yamabe problem; soliton; CR Yamabe flow. 
The flow approach has been introduced to study the CR Yamabe problem. The normalized CR Yamabe flow is defined as the evolution of the contact form $\theta(t)$ :

$$
\frac{\partial}{\partial t} \theta(t)=-\left(R_{\theta(t)}-\bar{R}_{\theta(t)}\right) \theta(t),\left.\theta(t)\right|_{t=0}=\theta,
$$

where $R_{\theta(t)}$ is the Tanaka-Webster scalar curvature of $\theta(t)$, and $\bar{R}_{\theta(t)}$ is the average of $R_{\theta(t)}$. The CR Yamabe flow has been studied by many authors. See [3, 5, 6, 7, 9, 14] and the references therein.

However, there has not been much work related to the CR Yamabe problem or $\mathrm{CR}$ Yamabe flow on noncomapct manifold. In [8, an existence result of the CR Yamabe problem on noncompact manifold was proved. As an analogue to the CR Yamabe flow on a compact CR manifold, one can define the (unnormalized) CR Yamabe flow as follows:

$$
\frac{\partial}{\partial t} \theta(t)=-R_{\theta(t)} \theta(t)
$$

where $R_{\theta(t)}$ is the Tanaka-Webster scalar curvature of $\theta(t)$. By using the maximum principles, we prove in section 4 a uniqueness theorem for the CR Yamabe flow (1.4) on noncompact manifold. See Theorem 4.6.

The CR Yamabe soliton is a self-similar solution to the (unnormalized) CR Yamabe flow. More precisely, $(M, \theta)$ is a CR Yamabe soliton if there exist an infinitesimal contact diffeomorphism $X$ and a constant $\mu$ such that

$$
\begin{aligned}
R_{\theta}+\frac{1}{2} \mathcal{L}_{X} \theta & =\mu, \\
\mathcal{L}_{X} J & =0 .
\end{aligned}
$$

Here $R_{\theta}$ is the Tanaka-Webster scalar curvature of $\theta$, and $\mathcal{L}_{X}$ is the Lie derivative with respect to $X$. It follows from ([1]) that, equivalently, $(M, \theta)$ is a CR Yamabe soliton if there exist a real-valued smooth function $f$ and a constant $\mu$ such that

$$
\begin{aligned}
R_{\theta}+\frac{1}{2} f_{0} & =\mu, \\
f_{\alpha \alpha}+\sqrt{-1} A_{\alpha \alpha} f & =0 \text { for all } \alpha=1,2, \ldots, n .
\end{aligned}
$$

Here $A_{\alpha_{\beta}}$ is the torsion of $\theta$, and $f$ is called potential function. Sometimes we also say that $(M, \theta, f)$ is a CR Yamabe soliton by specifying the potential function $f$. The CR Yamabe soliton is called shrinking if $\mu>0$, steady if $\mu=0$, and expanding if $\mu<0$ respectively.

The following lemma was proved in [1]: (see Lemma 3.2 in [1])

Proposition 1.2. A three-dimensional CR Yamabe soliton satisfies

$$
4 \Delta_{\theta} R_{\theta}+2 R_{\theta}\left(R_{\theta}-\mu\right)-\left(R_{\theta}\right)_{0} f-\left\langle\nabla_{\theta} R_{\theta}, J\left(\nabla_{\theta} f\right)\right\rangle_{\theta}=0 .
$$

By using Proposition 1.2. Cao, Chang and Chen proved in [1] that any compact three-dimensional CR Yamabe soliton must have constant Tanaka-Webster scalar curvature. See also [4 for another proof which is valid for all dimensions. In view of these results, one would like to study the CR Yamabe soliton on noncompact manifold. See [1, 2] for results related to the noncompact CR Yamabe soliton. In section 5, we study the noncompact CR Yamabe solitons. In particular, we are able to classify the CR Yamabe solitons on the Heisenberg group $\mathbb{H}^{n}$ equipped with the standard contact form. See Theorem 5.2 . 
This paper is organized as follows. In section 2, we recall some basic concepts in CR geometry. In section 3, we consider the CR Yamabe constant of conformal covering of $(M, \theta)$. In section 4 we prove a uniqueness theorem for the CR Yamabe flow (1.4) on noncompact manifold. Finally, in section 5, we study the CR Yamabe solitons on a complete noncompact CR manifold.

\section{Preliminaries and Notations}

Let $M$ be an orientable, real, $(2 n+1)$-dimensional manifold. A CR structure on $M$ is given by a complex $n$-dimensional subbundle $T_{1,0}$ of the complexified tangent bundle $\mathbb{C} T M$ of $M$, satisfying $T_{1,0} \cap T_{0,1}=\{0\}$, where $T_{0,1}=\bar{T}_{1,0}$. We assume the CR structure is integrable, that is, $T_{1,0}$ satisfies the formal Frobenius condition $\left[T_{1,0}, T_{0,1}\right] \subset T_{1,0}$. We set $G=\operatorname{Re}\left(T_{1,0} \oplus T_{0,1}\right)$, so that $G$ is a real $2 n$-dimensional subbundle of $T M$. Then $G$ carries a natural complex structure map: $J: G \rightarrow G$ given by $J(V+\bar{V})=\sqrt{-1}(V-\bar{V})$ for $V \in T_{1,0}$.

Let $E \subset T^{*} M$ denote the real line bundle $G^{\perp}$. Because we assume $M$ is orientable, and the complex structure $J$ induces an orientation on $G, E$ has a global nonvanishing section. A choice of such a 1 -form $\theta$ is called a pseudohermitian structure on $M$. Associated with each such $\theta$ is the real symmetric bilinear form $L_{\theta}$ on $G$ :

$$
L_{\theta}(V, W)=d \theta(V, J W), V, W \in G
$$

called the Levi-form of $\theta$. $L_{\theta}$ extends by complex linearity to $\mathbb{C} G$, and induces a Hermitian form on $T_{1,0}$, which we write

$$
L_{\theta}(V, \bar{W})=-\sqrt{-1} d \theta(V, \bar{W}), V, W \in T_{1,0}
$$

If $\theta$ is replaced by $\tilde{\theta}=f \theta, L_{\theta}$ changes conformally by $L_{\tilde{\theta}}=f L_{\theta}$. We will assume that $M$ is strictly pseudoconvex, that is, $L_{\theta}$ is positive definite for a suitable $\theta$. In this case, $\theta$ defines a contact structure on $M$, and we call $\theta$ a contact form. Then we define the volume form on $M$ as $d V_{\theta}=\theta \wedge d \theta^{n}$.

We can choose a unique $T$ called the characteristic direction such that $\theta(T)=1$, $d \theta(T, \cdot)=0$, and $T M=G \oplus \mathbb{R} T$. Then we can define a coframe $\left\{\theta, \theta^{1}, \theta^{2}, \cdots, \theta^{n}\right\}$ satisfying $\theta^{\alpha}(T)=0$, which is called admissible coframe. And its dual frame $\left\{T, Z_{1}, Z_{2}, \cdots, Z_{n}\right\}$ is called admissible frame. In this coframe, we have $d \theta=$ $\sqrt{-1} h_{\alpha \bar{\beta}} \theta^{\alpha} \wedge \theta^{\bar{\beta}}, h_{\alpha \bar{\beta}}$ is a Hermitian matrix.

The sub-Laplacian operator $\Delta_{\theta}$ is defined by

$$
\int_{M}\left(\Delta_{\theta} u\right) f d V_{\theta}=-\int_{M}\langle d u, d f\rangle_{\theta} d V_{\theta}
$$

for all smooth function $f$. Here $\langle,\rangle_{\theta}$ is the inner product induced by $L_{\theta}$. And we denote $\left|\nabla_{\theta} u\right|^{2}=\langle d u, d u\rangle_{\theta}$. Tanaka [15] and Webster [16] showed there is a natural connection in the bundle $T_{1,0}$ adapted to a pseudohermitian structure, which is called the Tanaka-Webster connection. To define this connection, we choose an admissible coframe $\left\{\theta^{\alpha}\right\}$ and dual frame $\left\{Z_{\alpha}\right\}$ for $T_{1,0}$. Then there are uniquely determined 1-forms $\omega_{\alpha \bar{\beta}}, \tau_{\alpha}$ on $M$, satisfying

$$
\begin{aligned}
d \theta^{\alpha} & =\omega_{\beta}^{\alpha} \wedge \theta^{\beta}+\theta \wedge \tau^{\alpha}, \\
d h_{\alpha \bar{\beta}} & =h_{\alpha \bar{\gamma}} \omega_{\bar{\beta}}^{\bar{\gamma}}+\omega_{\alpha}^{\gamma} h_{\beta \bar{\gamma}}, \\
\tau_{\alpha} \wedge \theta^{\alpha} & =0 .
\end{aligned}
$$


From the third equation, we can find $A_{\alpha \gamma}$, such that

$$
\tau_{\alpha}=A_{\alpha \gamma} \theta^{\gamma}
$$

and $A_{\alpha \gamma}=A_{\gamma \alpha}$. Here $A_{\alpha \gamma}$ is called the pseudohermitian torsion. With this connection, the covariant differentiation is defined by

$$
D Z_{\alpha}=\omega_{\alpha}^{\beta} \otimes Z_{\beta}, D Z_{\bar{\alpha}}=\omega_{\bar{\alpha}}^{\bar{\beta}} \otimes Z_{\bar{\beta}}, D T=0 .
$$

$\left\{\omega_{\beta}^{\alpha}\right\}$ are called connection 1-forms. For a smooth function $f$ on $M$, we write $f_{\alpha}=Z_{\alpha} f, f_{\bar{\alpha}}=Z_{\bar{\alpha}} f, f_{0}=T f$, so that $d f=f_{\alpha} \theta_{\alpha}+f_{\bar{\alpha}} \theta_{\bar{\alpha}}+f_{0} \theta$. The second covariant differential $D^{2} f$ is the 2 -tensor with components

$$
\begin{aligned}
& f_{\alpha \beta}=\overline{\bar{f}_{\bar{\alpha} \bar{\beta}}}=Z_{\beta} Z_{\alpha} f-\omega_{\alpha}^{\gamma}\left(Z_{\beta}\right) Z_{\gamma} f, f_{\alpha \bar{\beta}}={\overline{f_{\bar{\alpha} \beta}}}_{\bar{\alpha}}=Z_{\bar{\beta}} Z_{\alpha} f-\omega_{\alpha}^{\gamma}\left(Z_{\bar{\beta}}\right) Z_{\gamma} f, \\
& f_{0 \alpha}=\overline{\bar{f}_{0 \bar{\alpha}}}=Z_{\alpha} T f, f_{\alpha 0}={\overline{f_{\bar{\alpha} 0}}}_{\bar{\alpha}}=T Z_{\alpha} f-\omega_{\alpha}^{\gamma}(T) Z_{\gamma} f, f_{00}=T^{2} f .
\end{aligned}
$$

$h_{\alpha \bar{\beta}}$ and $h^{\alpha \bar{\beta}}$ are used to lower and raise the indices. The connections forms also satisfy

$d \omega_{\beta}^{\alpha}-\omega_{\beta}^{\gamma} \wedge \omega_{\gamma}^{\alpha}=\frac{1}{2} R_{\beta}^{\alpha} \rho \sigma \theta^{\rho} \wedge \theta^{\sigma}+\frac{1}{2} R_{\beta}^{\alpha} \bar{\rho} \bar{\sigma} \theta^{\bar{\rho}} \wedge \theta^{\bar{\sigma}}+R_{\beta}^{\alpha} \rho \bar{\sigma} \theta^{\rho} \wedge \theta^{\bar{\sigma}}+R_{\beta}^{\alpha} \rho 0 \theta^{\rho} \wedge \theta-R_{\beta}^{\alpha} \bar{\sigma} 0 \theta^{\bar{\sigma}} \wedge \theta$.

We call $R_{\beta \bar{\alpha} \rho \bar{\sigma}}$ the Tanaka-Webster curvature. Contractions of the Tanaka-Webster curvature yield the Tanaka-Webster Ricci curvature $R_{\rho \bar{\sigma}}=R_{\alpha \rho \bar{\sigma}}^{\alpha}$, or $R_{\rho \bar{\sigma}}=$ $h^{\alpha \bar{\beta}} R_{\alpha \bar{\beta} \rho \bar{\sigma}}$, and the Tanaka-Webster scalar curvature $R=h^{\rho \bar{\sigma}} R_{\rho \bar{\sigma}}$.

And we denote $d_{\theta}(\cdot, \cdot)$ to be the Carnot-Carathéodory distance with respect to $\theta$.

\section{The CR Yamabe COnstant of CONFormal COVering}

When the CR Yamabe constant $Y(M, \theta)<0$, any two contact forms with constant Tanaka-Webster scalar curvature are identical up to a scaling (see Theorem 7.1 in [10] or Theorem 1.3 in [5] for example). Hence, for a finite $k$-fold conformal covering $(\tilde{M}, \tilde{\theta})$ of $(M, \theta)$, we have

$$
Y(\tilde{M}, \tilde{\theta})=k^{\frac{1}{n+1}} Y(M, \theta) .
$$

On the other hand, when the CR Yamabe constant $Y(M, \theta)>0$, we cannot expect any similar explicit relations between $Y(\tilde{M}, \tilde{\theta})$ and $Y(M, \theta)$. One reason is that the uniqueness for the constant Tanaka-Webster scalar curvature mentioned above does not hold in general. However, we still have the following Aubin's Lemma for the CR case:

Lemma 3.1 (CR Aubin's Lemma). Let $(M, \theta)$ be a compact pseudoconvex $C R$ manifold of dimension $2 n+1$ with $Y(M, \theta)>0$, and $(\tilde{M}, \tilde{\theta})$ a nontrivial finite conformal covering of $(M, \theta)$. Then

$$
Y(M, \theta)<Y(\tilde{M}, \tilde{\theta}) .
$$

Proof. For the contact form $\theta$, we consider its lift $\tilde{\theta}$. Let $0<u \in C^{\infty}(\tilde{M})$ be a CR Yamabe minimizer with respect to $\tilde{\theta}$, i.e.

$$
-\left(2+\frac{2}{n}\right) \Delta_{\tilde{\theta}} u+R_{\tilde{\theta}} u=Y(\tilde{M}, \tilde{\theta}) u^{1+\frac{2}{n}} \text { on } \tilde{M} .
$$

Without loss of generality, we can assume

$$
\int_{\tilde{M}} u^{2+\frac{2}{n}} d V_{\tilde{\theta}}=1
$$


Let $\mathcal{G}$ be the deck transformation group for the normal covering $\tilde{M} \rightarrow M$. Consider its average

$$
v:=\sum_{\gamma \in \mathcal{G}} u \circ \gamma \text { on } \tilde{M}
$$

and define $0<v_{0} \in C^{\infty}(M)$ to be the function whose lift to $\tilde{M}$ is $v$. Then

$$
Y(M, \theta) \leq E_{(M, \theta)}\left(v_{0}\right)=\frac{\int_{M}\left(\left(2+\frac{2}{n}\right)\left|\nabla_{\theta} v_{0}\right|^{2}+R_{\theta} v_{0}^{2}\right) d V_{\theta}}{\left(\int_{M} v_{0}^{2+\frac{2}{n}} d V_{\theta}\right)^{\frac{n}{n+1}}}
$$

Since $(\tilde{M}, \tilde{\theta})$ is a finite $k$-fold conformal covering of $(M, \theta)$, we have

$$
\int_{M} v_{0}^{2+\frac{2}{n}} d V_{\theta}=\frac{1}{k} \int_{\tilde{M}} v^{2+\frac{2}{n}} d V_{\tilde{\theta}}
$$

and

$$
\begin{aligned}
& \int_{M}\left(\left(2+\frac{2}{n}\right)\left|\nabla_{\theta} v_{0}\right|^{2}+R_{\theta} v_{0}^{2}\right) d V_{\theta} \\
& =\frac{1}{k} \int_{\tilde{M}}\left(\left(2+\frac{2}{n}\right)\left|\nabla_{\tilde{\theta}} v\right|^{2}+R_{\tilde{\theta}} v^{2}\right) d V_{\tilde{\theta}} \\
& =\frac{1}{k} \int_{\tilde{M}}\left(-\left(2+\frac{2}{n}\right) \Delta_{\tilde{\theta}} v+R_{\tilde{\theta}} v\right) v d V_{\tilde{\theta}} \\
& =\frac{1}{k} \sum_{\gamma \in \mathcal{G}} \int_{\tilde{M}}\left(-\left(2+\frac{2}{n}\right) \Delta_{\tilde{\theta}}(u \circ \gamma)+R_{\tilde{\theta}}(u \circ \gamma)\right) v d V_{\tilde{\theta}} \\
& =\frac{1}{k} Y(\tilde{M}, \tilde{\theta}) \int_{\tilde{M}} \sum_{\gamma \in \mathcal{G}}(u \circ \gamma)^{1+\frac{2}{n}} v d V_{\tilde{\theta}}
\end{aligned}
$$

where we have used (3.1) and (3.3). Substituting (3.5) and (3.6) into (3.4), we get

$$
Y(M, \theta) \leq k^{-\frac{1}{n+1}} Y(\tilde{M}, \tilde{\theta}) \frac{\int_{\tilde{M}} \sum_{\gamma \in \mathcal{G}}(u \circ \gamma)^{1+\frac{2}{n}} v d V_{\tilde{\theta}}}{\left(\int_{\tilde{M}} v^{2+\frac{2}{n}} d V_{\tilde{\theta}}\right)^{\frac{n}{n+1}}} .
$$

By Hölder's inequality and the inequality $\left(\sum_{i=1}^{k} a_{i}^{p}\right)^{\frac{1}{p}}<\sum_{i=1}^{k} a_{i}$ for $k \geq 2, p>1$, $a_{i}>0$, we can compute

$$
\begin{aligned}
\int_{\tilde{M}} \sum_{\gamma \in \mathcal{G}}(u \circ \gamma)^{1+\frac{2}{n}} v d V_{\tilde{\theta}} & \leq \int_{\tilde{M}}\left(\sum_{\gamma \in \mathcal{G}}(u \circ \gamma)^{2+\frac{2}{n}}\right)^{\frac{1}{n+1}}\left(\sum_{\gamma \in \mathcal{G}}(u \circ \gamma)^{\frac{n+1}{n}}\right)^{\frac{n}{n+1}} v d V_{\tilde{\theta}} \\
& <\int_{\tilde{M}}\left(\sum_{\gamma \in \mathcal{G}}(u \circ \gamma)^{2+\frac{2}{n}}\right)^{\frac{1}{n+1}}\left(\sum_{\gamma \in \mathcal{G}} u \circ \gamma\right) v d V_{\tilde{\theta}} \\
& =\int_{\tilde{M}}\left(\sum_{\gamma \in \mathcal{G}}(u \circ \gamma)^{2+\frac{2}{n}}\right)^{\frac{1}{n+1}} v^{2} d V_{\tilde{\theta}} \\
& \leq\left(\int_{\tilde{M}} v^{2+\frac{2}{n}} d V_{\tilde{\theta}}\right)^{\frac{n}{n+1}}\left(\int_{\tilde{M}} \sum_{\gamma \in \mathcal{G}}(u \circ \gamma)^{2+\frac{2}{n}} d V_{\tilde{\theta}}\right)^{\frac{1}{n+1}} .
\end{aligned}
$$


It follows from (3.2) that

$$
\int_{\tilde{M}} \sum_{\gamma \in \mathcal{G}}(u \circ \gamma)^{2+\frac{2}{n}} d V_{\tilde{\theta}}=k \int_{\tilde{M}} u^{2+\frac{2}{n}} d V_{\tilde{\theta}}=k .
$$

Now the assertion follows from combining (3.7)-(3.9).

For a noncompact CR manifold $(X, \theta)$, the CR Yamabe constant of $(X, \theta)$ is defined by

$$
Y(X, \theta)=\inf \left\{E_{(X, \theta)}(u) \mid 0<u \in C_{c}^{\infty}(X)\right\} .
$$

Here, $C_{c}^{\infty}(X)$ is the space of all compactly supported smooth functions in $X$.

The argument of Jerison and Lee in Theorem 1.1 is still valid for any noncompact manifold:

$$
Y(X, \theta) \leq Y\left(S^{2 n+1}, \theta_{S^{2 n+1}}\right) .
$$

We have the following definition:

Let $G$ be an infinite group and $H$ a subgroup of $G$ with infinite index. Let $\left\{G_{i}\right\}_{i \geq 1}$ be an infinite sequence of subgroups of $G$. We call $\left\{G_{i}\right\}_{i \geq 1}$ a descending chain of finite index subgroups tending to $H$ if

(i) each $G_{i}$ is a finite index subgroup of $G$ with $G_{i} \supset H$;

(ii) $G=G_{1} \supsetneq G_{2} \supsetneq \cdots \supsetneq G_{i} \supsetneq G_{i+1} \supsetneq \cdots$; and

(iii) $\bigcap_{i=1}^{\infty} G_{i}=H$.

The following theorem corresponds to an analogue of Lemma 3.1 for the CR Yamabe constants of infinite conformal coverings. We identify each $\pi_{1}\left(M_{k}\right)$ and $\pi_{1}\left(M_{\infty}\right)$ with their projections to $\pi_{1}(M)$ in the following theorem.

Theorem 3.2. Let $(M, \theta)$ be a compact pseudoconvex $C R$ manifold of dimension $2 n+1$ with $Y(M, \theta)>0$. Let $\left(M_{\infty}, \theta_{\infty}\right) \rightarrow(M, \theta)$ be an infinite conformal covering such that $\pi_{1}(M)$ has a descending chain of finite index subgroups tending to $\pi_{1}\left(M_{\infty}\right)$. Then

$$
Y(M, \theta)<Y\left(M_{\infty}, \theta_{\infty}\right) .
$$

Proof. Let $0<u \in C^{\infty}(M)$ be a CR Yamabe minimizer with respect to $\theta$, i.e. $E_{(M, \theta)}(u)=Y(M, \theta)$. By replacing $\theta$ by

$$
\tilde{\theta}=\frac{u^{\frac{2}{n}} \theta}{\left(\int_{M} u^{2+\frac{2}{n}} d V_{\theta}\right)^{\frac{1}{n+1}}}
$$

we may assume that

$$
R_{\theta}=Y(M, \theta) \text { and } \int_{M} d V_{\theta}=1 .
$$

Consider the lift $\theta_{\infty}$ of $\theta$ to $M_{\infty}$. Note that

$$
R_{\theta_{\infty}}=R_{\theta}=Y(M, \theta)>0 .
$$

The Folland-Stein embedding

$$
S_{1}^{2}\left(M_{\infty}, \theta_{\infty}\right) \hookrightarrow L^{2+\frac{2}{n}}\left(M_{\infty}, \theta_{\infty}\right)
$$


combined with (3.12) implies that $Y\left(M_{\infty}, \theta_{\infty}\right)>0$. To see this, it follows from the Folland-Stein embedding that there exists a uniform constant $C_{0}>0$ depending only on $\left(M_{\infty}, \theta_{\infty}\right)$ such that

$$
\left(\int_{M_{\infty}} \varphi^{2+\frac{2}{n}} d V_{\theta_{\infty}}\right)^{\frac{n}{n+1}} \leq C_{0} \int_{M_{\infty}}\left(\left|\nabla_{\theta_{\infty}} \varphi\right|^{2}+\varphi^{2}\right) d V_{\theta_{\infty}}
$$

for any $\varphi \in C_{c}^{\infty}\left(M_{\infty}\right)$. Thus, for any $\varphi \in C_{c}^{\infty}\left(M_{\infty}\right)$, we have

$$
\begin{aligned}
E_{\left(M_{\infty}, \theta_{\infty}\right)}(\varphi) & =\frac{\int_{M_{\infty}}\left(\left(2+\frac{2}{n}\right)\left|\nabla_{\theta_{\infty}} \varphi\right|^{2}+R_{\theta_{\infty}} \varphi^{2}\right) d V_{\theta_{\infty}}}{\left(\int_{M_{\infty}} \varphi^{2+\frac{2}{n}} d V_{\theta_{\infty}}\right)^{\frac{n}{n+1}}} \\
& \geq \min \left\{2+\frac{2}{n}, Y(M, \theta)\right\} \frac{\int_{M_{\infty}}\left(\left|\nabla_{\theta_{\infty}} \varphi\right|^{2}+\varphi^{2}\right) d V_{\theta_{\infty}}}{\left(\int_{M_{\infty}} \varphi^{2+\frac{2}{n}} d V_{\theta_{\infty}}\right)^{\frac{n}{n+1}}} \\
& \geq C_{0} \min \left\{2+\frac{2}{n}, Y(M, \theta)\right\}
\end{aligned}
$$

by (3.12) and (3.13). This implies that $Y\left(M_{\infty}, \theta_{\infty}\right) \geq C_{0} \min \left\{2+\frac{2}{n}, Y(M, \theta)\right\}>0$, as we claim.

For the covering $M_{\infty} \rightarrow M$, there exist base points $p_{\infty} \in M_{\infty}, p_{1} \in M$ and the projection map $\mathcal{P}:\left(M_{\infty}, p_{\infty}\right) \rightarrow\left(M, p_{1}\right)$ such that $\pi_{1}\left(M_{\infty}\right)=\pi_{1}\left(M_{\infty}, p_{\infty}\right)$ and $\pi_{1}(M)=\pi_{1}\left(M, p_{1}\right)$. Since $\pi_{1}(M)$ has a descending chain of finite index subgroups tending to $\pi_{1}\left(M_{\infty}\right)$, for each $k \geq 2$, there exists a finite covering $P_{k}:\left(M_{k}, p_{k}\right) \rightarrow$ $\left(M, p_{1}\right)$ satisfying $\bigcap_{k=1}^{\infty} \pi_{1}\left(M_{k}\right)=\pi_{1}\left(M_{\infty}\right)\left(M_{1}=M\right.$ and $\left.\pi_{1}\left(M_{k}\right)=\pi_{1}\left(M_{k}, p_{k}\right)\right)$ and the following:

(i) $M_{\infty}$ is an infinite covering $\mathcal{P}_{k}:\left(M_{\infty}, p_{\infty}\right) \rightarrow\left(M_{k}, p_{k}\right)$ of each $M_{k}$;

(ii) $M_{k+1}$ is a non-trivial finite covering $P_{k, k+1}:\left(M_{k+1}, p_{k+1}\right) \rightarrow\left(M_{k}, p_{k}\right)$ of each $M_{k}$ for $k \geq 1$.

Here we identify $\pi_{1}\left(M_{\infty}\right)$ and $\pi_{1}\left(M_{k}\right)$ with their projections to $\pi_{1}\left(M, p_{1}\right)$. Denote the lifting of $\theta$ to $M_{k}$ by $\theta_{k}$. From Theorem 1.1(i) and Lemma 3.1, we have

$Y(M, \theta)<Y\left(M_{2}, \theta_{2}\right)<\cdots<Y\left(M_{k}, \theta_{k}\right)<Y\left(M_{k+1}, \theta_{k+1}\right)<\cdots \leq Y\left(S^{2 n+1}, \theta_{S^{2 n+1}}\right)$,

and hence the limit of $\left\{Y\left(M_{k},\left[\theta_{k}\right]\right)\right\}_{k \geq 1}$ always exists. Therefore, by taking a suitable subsequence $\left\{Y\left(M_{k_{j}},\left[\theta_{k_{j}}\right]\right)\right\}_{j \geq 1}$ if necessary, it suffices to consider only the subsequence for the proof. Throughout this paper, we always assume that $\left(M_{k_{1}}, \theta_{k_{1}}\right)=(M, \theta)$ for such a subsequence $\left\{\left(M_{k_{j}}, \theta_{k_{j}}\right)\right\}_{j \geq 1}$.

For each $k \geq 1$, we set

$$
\mathcal{D}_{k}=\left\{x \in M_{\infty} \mid d_{\theta_{\infty}}\left(x, p_{\infty}\right)<d_{\theta_{\infty}}\left(x, q_{\infty}\right) \text { for all } q_{\infty} \in \mathcal{P}_{k}^{-1}\left(p_{k}\right)-\left\{p_{\infty}\right\}\right\} .
$$

Here $d_{\theta_{\infty}}(\cdot, \cdot)$ is the Carnot-Carathéodory distance function with respect to $\theta_{\infty}$. Since $M_{k}$ is compact and $\theta_{\infty}$ is the lifting of $\theta$ on $M=M_{1}$ to $M_{\infty}$, the infimum

$$
\inf \left\{d_{\theta_{\infty}}\left(q_{\infty}, \tilde{q}_{\infty}\right) \mid q_{\infty} \neq \tilde{q}_{\infty}, q_{\infty}, \tilde{q}_{\infty} \in \mathcal{P}_{k}^{-1}\left(p_{k}\right)\right\}
$$

is strictly positive. Hence, there exists a finite subset $\mathcal{A}_{k} \subset \mathcal{P}_{k}^{-1}\left(p_{k}\right)-\left\{p_{\infty}\right\}$ for each $k \geq 1$ such that

$$
\mathcal{D}_{k}=\left\{x \in M_{\infty} \mid d_{\theta_{\infty}}\left(x, p_{\infty}\right)<d_{\theta_{\infty}}\left(x, q_{\infty}\right) \text { for all } q_{\infty} \in \mathcal{A}_{k}\right\} .
$$


Therefore, $\mathcal{D}_{k}$ is an open set, and especially a fundamental domain containing $p_{\infty}$ for the covering $M_{\infty} \rightarrow M_{k}$ such that

$$
\mathcal{D}_{1} \subset \mathcal{D}_{2} \subset \cdots \subset \mathcal{D}_{k} \subset \mathcal{D}_{k+1} \subset \cdots M_{\infty}, \bigcup_{k \geq 1} \mathcal{D}_{k}=M_{\infty} .
$$

From (3.15), by taking a suitable subsequence if necessary, we can assume the following:

(iii) For all $k \geq 1$, the closure $\widehat{M}_{k}:=\overline{\mathcal{D}}_{k} \subset M_{\infty}$ satisfies $d_{\theta_{\infty}}\left(\widehat{M}_{k}, \partial \widehat{M}_{k+1}\right) \geq 1$.

By (iii), we can take a sequence $\left\{\Omega_{k}\right\}_{k \geq 2}$ of domains in $M_{\infty}$ with smooth boundary $\partial \bar{\Omega}_{k}$ satisfying

$$
\widehat{M}_{k-1} \subset \Omega_{k} \subset \widehat{M}_{k} \text { for } k \geq 2 .
$$

We will denote $\widehat{M}:=\widehat{M}_{1}$.

When $Y\left(M_{\infty}, \theta_{\infty}\right)=Y\left(S^{2 n+1}, \theta_{S^{2 n+1}}\right)$, it follows from (3.14), Theorem 1.1(i) and Lemma 3.1 that

$$
Y(M, \theta)<Y\left(M_{2}, \theta_{2}\right) \leq Y\left(S^{2 n+1}, \theta_{S^{2 n+1}}\right)=Y\left(M_{\infty}, \theta_{\infty}\right),
$$

which proves (3.11). Hence, we may assume $Y\left(M_{\infty}, \theta_{\infty}\right)<Y\left(S^{2 n+1}, \theta_{S^{2 n+1}}\right)$ by (3.10).

By definition of $Y\left(M_{\infty}, \theta_{\infty}\right)$ and the condition (iii), there exists $k_{0} \in \mathbb{N}$ such that

$$
Q_{k}:=\inf _{\varphi \in C_{c}^{\infty}\left(\Omega_{k}\right)} E_{\left(M_{\infty}, \theta_{\infty}\right)}(\varphi)<Y\left(S^{2 n+1}, \theta_{S^{2 n+1}}\right) \text { for all } k \leq k_{0}
$$

and that

$$
Q_{k_{0}}>Q_{k_{0}+1}>\cdots>Q_{k_{0}+i}>Q_{k_{0}+i+1}>\cdots, \lim _{k \rightarrow \infty} Q_{k}=Y\left(M_{\infty}, \theta_{\infty}\right) .
$$

By combining the inequality $Q_{k}<Y\left(S^{2 n+1}, \theta_{S^{2 n+1}}\right)$ with the same argument of Jerison and Lee in proving Theorem 1.1(ii) for the compact manifolds, the CR Yamabe problem of Dirichlet-type can be solved for each $\left(\bar{\Omega}_{k},\left.\theta_{\infty}\right|_{\bar{\Omega}_{k}}\right)$. Namely, there exits $\psi_{k} \in C^{\infty}\left(\bar{\Omega}_{k}\right)$ such that

$$
E_{\left(M_{\infty}, \theta_{\infty}\right)}\left(\psi_{k}\right)=Q_{k}, \psi_{k}>0 \text { in } \Omega_{k} \text { and } \psi_{k}=0 \text { on } \partial \bar{\Omega}_{k} .
$$

We denote the zero extension of $\psi_{k}$ to $M_{\infty}$ also by $\psi_{k} \in C^{0,1}\left(M_{\infty}\right) \cap S_{1}^{2}\left(M_{\infty}, \theta_{\infty}\right)$. Because $\left.\psi_{k}\right|_{\partial \bar{\Omega}_{k}}=0, \psi_{k}$ can also be regarded as a function on the compact manifold $M_{k}$. Then

$$
Y\left(M_{k}, \theta_{k}\right) \leq E_{\left(M_{k}, \theta_{k}\right)}\left(\psi_{k}\right)=E_{\left(M_{\infty}, \theta_{\infty}\right)}\left(\psi_{k}\right)=Q_{k}
$$

and hence

$$
\limsup _{k \rightarrow \infty} Y\left(M_{k}, \theta_{k}\right) \leq \lim _{k \rightarrow \infty} Q_{k}=Y\left(M_{\infty}, \theta_{\infty}\right) .
$$

From Lemma 3.1 we obtain

$$
Y(M, \theta)<Y\left(M_{2}, \theta_{2}\right) \leq \lim _{k \rightarrow \infty} Y\left(M_{k}, \theta_{k}\right)=\limsup _{k \rightarrow \infty} Y\left(M_{k}, \theta_{k}\right) \leq Y\left(M_{\infty}, \theta_{\infty}\right) .
$$

This completes the proof of (3.11). 


\section{The CR YAmabe Flow ON COMPlete NONCOMPACT MANIFOLDS}

Let $(M, \theta)$ be a smooth, strictly pseudoconvex $(2 n+1)$-dimensional complete noncompact CR manifold. If we write $\theta(t)=u^{\frac{2}{n}} \theta$ for some $u=u(t)$, then it follows from (1.2) that the CR Yamabe flow (1.4) reduces to

$$
\frac{\partial u}{\partial t}=(n+1) u^{-\frac{2}{n}} \Delta_{\theta} u-\frac{n}{2} R_{\theta} u^{1-\frac{2}{n}}
$$

from which we obtain

$$
\frac{\partial}{\partial t}\left(u^{\frac{n+2}{n}}\right)=\frac{(n+1)(n+2)}{n}\left(\Delta_{\theta} u-\frac{n}{2 n+2} R_{\theta} u\right) .
$$

Now we will establish two maximum principles which hold on complete noncompact $\mathrm{CR}$ manifolds. By using the maximum principles, we prove a uniqueness theorem for the CR Yamabe flow (1.4). To do this, we follow the idea of Ma and An in [12]. First we give two definitions.

Definition 4.1. We say that a complete noncompact $C R$ manifold $(M, \theta)$ satisfies Condition $A$, if there exist a constant $A$ and a smooth positive function $f$ such that $f \rightarrow \infty$ near infinity and

$$
\left|\nabla_{\theta} f\right| \leq A \quad \text { and } \Delta_{\theta} f \leq A \text { on } M .
$$

Definition 4.2. Let $t \in[0, T]$, we say $(M, \theta(t))$ satisfy Condition $B$, if there exist a positive constant $B$ and a smooth positive function $g$ such that

$$
-\frac{\partial}{\partial t} g+\Delta_{\theta} g \leq B, \text { on } M \times[0, T]
$$

and for any constant $C>0, x_{0} \in M$, there is a positive constant $d_{0}$ satisfying $g(x, t)>C$ for $d_{\theta(t)}\left(x_{0}, x\right)>d_{0}$ for all $t \in[0, T]$.

Example 4.3. We claim that the Heisenberg group $\mathbb{H}^{n}$ satisfies Condition $A$ and Condition $B$ above. Recall that the Heisenberg group $\mathbb{H}^{n}$ is a Lie group whose underlying manifold is $\mathbb{C}^{n} \times \mathbb{R}$ with coordinates $(z, t)=\left(z^{1}, z^{2}, \cdots, z^{n}, t\right)$. The contact form of $\mathbb{H}^{n}$ is

$$
\theta_{0}=d t+\sqrt{-1} \sum_{\alpha=1}^{n}\left(z^{\alpha} d \bar{z}^{\alpha}-\bar{z}^{\alpha} d z^{\alpha}\right)
$$

Then the dual frame $\left\{Z_{\alpha}\right\}$ for $T^{1,0}$ defined in section 2 are given by

$$
Z_{\alpha}=\frac{1}{\sqrt{2}}\left(\frac{\partial}{\partial z^{\alpha}}+\sqrt{-1} \bar{z}^{\alpha} \frac{\partial}{\partial t}\right) \quad \text { for } 1 \leq \alpha \leq n .
$$

And $Z_{\bar{\alpha}}=\bar{Z}_{\alpha}$. If we define $f(z, t)=\left(|z|^{4}+t^{2}\right)^{p}$ for $(z, t) \in \mathbb{H}^{n}$, where $0<p \leq 1 / 4$, then $f \rightarrow \infty$ near infinity, and

$$
\begin{aligned}
Z_{\alpha} f & =\frac{p\left(|z|^{2}+\sqrt{-1} t\right) \bar{z}_{\alpha}}{\sqrt{2}\left(|z|^{4}+t^{2}\right)^{1-p}}, \\
Z_{\bar{\alpha}} Z_{\alpha} f & =\frac{p\left(|z|^{2}+\sqrt{-1} t\right)}{2\left(|z|^{4}+t^{2}\right)^{1-p}}+\frac{p\left|z_{\alpha}\right|^{2}}{\left(|z|^{4}+t^{2}\right)^{1-p}}-\frac{p(1-p)\left|z_{\alpha}\right|^{2}}{2\left(|z|^{4}+t^{2}\right)^{1-p}}
\end{aligned}
$$

for all $1 \leq \alpha \leq n$. This implies that

$$
\left|\nabla_{\theta_{0}} f\right|=\sum_{\alpha=1}^{n} Z_{\alpha} f Z_{\bar{\alpha}} f \leq A \quad \text { and } \quad \Delta_{\theta_{0}} f=\sum_{\alpha=1}^{n}\left(Z_{\bar{\alpha}} Z_{\alpha}+Z_{\alpha} Z_{\bar{\alpha}}\right) f \leq A
$$


for some uniform constant $A$, since $0<p \leq 1 / 4$. Thus the Heisenberg group $\mathbb{H}^{n}$ is an example satisfies Condition $A$, and also satisfies Condition $B$ by fixing the contact form $\theta(t)=\theta$ and the function $g=f$.

Now we prove the following weak maximum principle:

Theorem 4.4. Let $(M, \theta)$ be a complete noncompact $C R$ manifold satisfying Condition A. Assume $u \in C^{2}(M \times[0, T])$ satisfies the following evolution equation:

$$
\begin{aligned}
\frac{\partial}{\partial t} u & =\Delta_{\theta} u+F(D u, u, x, t), \quad \text { on } M \times[0, T], \\
|u| & \leq C, \quad \text { on } M \times[0, T], \\
u(x, 0) & \leq 0, \quad \text { on } M, \\
F(D u, u, x, t) & \leq C u(x, t), \text { for } u(x, t)>0 .
\end{aligned}
$$

Here $C>0$ is a fixed constant. Then we have $u \leq 0$ on $M \times[0, T]$.

Proof. We argue by contradiction. Assume that there is a point $\left(x_{0}, t_{0}\right) \in M \times[0, T]$ such that

$$
u\left(x_{0}, t_{0}\right)>0 .
$$

We denote $F(x, t)=F(D u, u, x, t)$. Let $\psi_{\lambda}(x, t)=e^{\lambda t}(f(x)+A t+1)$, where $\lambda$ is a constant chosen to satisfy $\lambda>C$. Then we have

$$
\begin{aligned}
\frac{\partial \psi_{\lambda}}{\partial t} & =\lambda \psi_{\lambda}+e^{\lambda t} A \\
& \geq \lambda \psi_{\lambda}+\Delta_{\theta} \psi_{\lambda} .
\end{aligned}
$$

Let $\varphi_{\lambda}=\frac{1}{\psi_{\lambda}}$, i.e. $0 \leq \varphi_{\lambda} \leq 1$. From which we get

$$
\begin{aligned}
\frac{\partial \varphi_{\lambda}}{\partial t}=-\psi_{\lambda}^{-2} \frac{\partial \psi_{\lambda}}{\partial t} & \leq-\psi_{\lambda}^{-2}\left(\lambda \psi_{\lambda}+\Delta_{\theta} \psi_{\lambda}\right) \\
& =-\lambda \varphi_{\lambda}-\psi_{\lambda}^{-2} \Delta_{\theta} \psi_{\lambda} .
\end{aligned}
$$

And

$$
\begin{aligned}
\Delta_{\theta} \varphi_{\lambda} & =-\psi_{\lambda}^{-2} \Delta_{\theta} \psi_{\lambda}+2 \psi_{\lambda}^{-3}\left|\nabla \psi_{\lambda}\right|^{2} \\
& =-\psi_{\lambda}^{-2} \Delta_{\theta} \psi_{\lambda}+2 \varphi_{\lambda}^{-1}\left|\nabla_{\theta} \varphi_{\lambda}\right|^{2} .
\end{aligned}
$$

From the above, we can deduce that

$$
-\psi_{\lambda}^{-2} \Delta_{\theta} \psi_{\lambda}=\Delta_{\theta} \varphi_{\lambda}-2 \varphi_{\lambda}^{-1}\left|\nabla_{\theta} \varphi_{\lambda}\right|^{2} .
$$

Then we have

$$
\frac{\partial \varphi_{\lambda}}{\partial t} \leq \Delta_{\theta} \varphi_{\lambda}-2 \varphi_{\lambda}^{-1}\left|\nabla_{\theta} \varphi_{\lambda}\right|^{2}-\lambda \varphi_{\lambda} \text { on } M .
$$

Set $v=\varphi_{\lambda} u$. Then we have $v\left(x_{0}, t_{0}\right)=\varphi_{\lambda}\left(x_{0}, t_{0}\right) u\left(x_{0}, t_{0}\right)>0$. Since $|u| \leq C$ and $0<\varphi_{\lambda} \leq 1$, we can assume

$$
a=\sup _{M \times[0, T]} v>0
$$

We denote

$$
D=\left\{x \in M: d_{\theta}\left(x_{0}, x\right) \leq \frac{C_{1}}{a}\right\} .
$$

Here $C_{1}$ is a positive constant. Since $\varphi_{\lambda}$ tends to 0 near infinity, we can choose $C_{1}$ large enough such that

$$
v(x, t)<a, \text { for }(x, t) \notin D \times[0, T] .
$$


Since $D \times[0, t]$ is compact, we can find an interior point $\left(x_{1}, t_{1}\right) \in D \times[0, T]$ such that

$$
\sup _{M \times[0, T]} v(x, t)=v\left(x_{1}, t_{1}\right)=a .
$$

At the point $\left(x_{1}, t_{1}\right)$, we have

$$
\frac{\partial v}{\partial t}-\Delta_{\theta} v \geq 0
$$

and

$$
\nabla_{\theta} v=0
$$

Then by (4.4), we have

$$
\begin{aligned}
\frac{\partial v}{\partial t}-\Delta_{\theta} v & =-2 \varphi_{\lambda}^{-1}\left\langle\nabla_{\theta} \varphi_{\lambda}, \nabla_{\theta} v\right\rangle+\varphi_{\lambda} F(x, t)+\left(\frac{\partial \varphi_{\lambda}}{\partial t}-\Delta_{\theta} \varphi_{\lambda}+\frac{2}{\varphi_{\lambda}}\left|\nabla_{\theta} \varphi_{\lambda}\right|^{2}\right) u \\
& \leq-2 \varphi_{\lambda}^{-1}\left\langle\nabla_{\theta} \varphi_{\lambda}, \nabla_{\theta} v\right\rangle+\varphi_{\lambda} F(x, t)-\lambda \varphi_{\lambda} u
\end{aligned}
$$

Now at the point $\left(x_{1}, t_{1}\right)$, we obtain

$$
0 \leq \varphi_{\lambda} F-\lambda \varphi_{\lambda} u \text {. }
$$

Thus

$$
F\left(x_{1}, t_{1}\right) \geq \lambda u\left(x_{1}, t_{1}\right)>C u\left(x_{1}, t_{1}\right),
$$

which is absurd by our assumption.

Now we follow the idea of Ma and An [12] to prove the next maximum principle.

Theorem 4.5. Let $(M, \theta)$ be a complete noncompact $C R$ manifold. And $(M, \theta(t))$ satisfies Condition $B$ on $M \times[0, T]$. Assume $u \in C^{2}(M \times[0, T])$ satisfies the following evolution equation:

$$
\begin{aligned}
\frac{\partial}{\partial t} u-\Delta_{\theta} u \leq 0, & \text { on } M \times[0, T], \\
u(0)=u_{0} \leq C, & \text { on } M .
\end{aligned}
$$

Then $u \leq C$ on $M \times[0, T]$.

Proof. Without loss of generality, we can assume $C>0$. And let $s>0$ be the largest time such that

$$
C \leq \sup _{M \times[0, T]} u \leq 2 C
$$

We define $w$ by

$$
w=u-\epsilon\left(g+C_{1} t\right),
$$

where $C_{1} \geq B$ is a fixed constant, and $\epsilon>0$. We choose $A$ such that

$$
\epsilon A>C .
$$

Then we have

$$
\begin{aligned}
\frac{\partial}{\partial t} w-\Delta_{\theta} w & =\frac{\partial}{\partial t} u-\Delta_{\theta} u-\epsilon\left(\frac{\partial}{\partial t} g-\Delta_{\theta} g+C_{1}\right) \\
& \leq \frac{\partial}{\partial t} u-\Delta_{\theta} u+\epsilon\left(B-C_{1}\right) \leq 0 .
\end{aligned}
$$

Since $w \leq 2 C-\epsilon A<C$ for $d_{\theta}\left(x_{0}, x\right)>d_{0}$. Hence, $w$ can attain its supremum at a point $x_{1} \in M$. Then at point $\left(x_{1}, t\right)$, we have

$$
\Delta_{\theta} w \leq 0
$$


From which we get

$$
\frac{\partial}{\partial t}\left(\sup _{M} w(t)\right) \leq 0
$$

Therefore,

$$
u-\epsilon\left(g+C_{1} t\right) \leq \sup _{M} w(t) \leq \sup _{M} w(0) \leq C .
$$

Let $\epsilon$ tends to 0 in (4.5), we obtain for any $t \leq s$,

$$
u(x, t) \leq C .
$$

Then by iteration, we get the conclusion that $u(x, t) \leq C$ on $M \times[0, T]$.

Next we prove the following uniqueness theorem of the CR Yamabe flow (1.4) on complete noncompact CR manifolds.

Theorem 4.6. Let $(M, \theta)$ be a complete noncompact $C R$ manifold. Suppose that $u$ is the solution of the $C R$ Yamabe flow (4.2) such that $\left(M, \theta(t)=u^{\frac{2}{n}} \theta\right)$ satisfies Condition $B$ on $M \times[0, T]$. If $u \in C^{2}(M \times[0, T])$ and $0<c \leq u \leq C$ on $M \times[0, T]$, then $u$ is the unique solution of the CR Yamabe flow (4.2).

Proof. The CR Yamabe flow (4.2) is given by

$$
u^{\frac{2}{n}} \frac{\partial u}{\partial t}=(n+1)\left(\Delta_{\theta} u-\frac{n}{2(n+1)} R_{\theta} u\right), u(0)=u_{0}>0 .
$$

Now we argue by contradiction. Suppose that there exists two positive solutions $u, v$ of this initial value problem (4.6). We define

$$
w=u-v .
$$

Then, in $M \times[0, T]$, we have

$$
\begin{aligned}
(n+1)\left(\Delta_{\theta} w-\frac{n}{2(n+1)} R_{\theta} w\right) & =u^{\frac{2}{n}} \frac{\partial u}{\partial t}-v^{\frac{2}{n}} \frac{\partial v}{\partial t} \\
& =u^{\frac{2}{n}} \frac{\partial w}{\partial t}+\left(u^{\frac{2}{n}}-v^{\frac{2}{n}}\right) \frac{\partial v}{\partial t} \\
& =u^{\frac{2}{n}} \frac{\partial w}{\partial t}+\left(\int_{0}^{1} \frac{d}{d z}(z u+(1-z) v)^{\frac{2}{n}} d z\right) \frac{\partial v}{\partial t} \\
& =u^{\frac{2}{n}} \frac{\partial w}{\partial t}+\left(\int_{0}^{1} \frac{2}{n}(z u+(1-z) v)^{\frac{2-n}{n}}(u-v) d z\right) \frac{\partial v}{\partial t} \\
& =u^{\frac{2}{n}} \frac{\partial w}{\partial t}+\left(\int_{0}^{1}(z u+(1-z) v)^{\frac{2-n}{n}} d z\right) \frac{2}{n} w \frac{\partial v}{\partial t} .
\end{aligned}
$$


It follows from (4.7) that

$$
\begin{aligned}
\frac{\partial w}{\partial t} & =\frac{n+1}{u^{\frac{2}{n}}} \Delta_{\theta} w-\frac{n}{2} R_{0} w u^{-\frac{2}{n}} \\
& -\frac{2}{n} u^{-\frac{2}{n}} \frac{\partial v}{\partial t} w \int_{0}^{1}(z u+(1-z) v)^{\frac{2-n}{n}} d z \\
& =\frac{n+1}{u^{\frac{2}{n}}} \Delta_{\theta} w-\left(\frac{2}{n u^{\frac{2}{n}}} \frac{\partial v}{\partial t} \int_{0}^{1}(z u+(1-z) v)^{\frac{2-n}{n}} d z+\frac{2}{n} \frac{R_{\theta}}{u^{\frac{2}{n}}}\right) w \\
& =a \Delta_{\theta} w-b w, \quad \text { on } M \times[0, T], \\
w(0) & \equiv 0 .
\end{aligned}
$$

Here

$$
a=\frac{n+1}{u^{\frac{2}{n}}}>0 \text { and } b=\frac{2}{n u^{\frac{2}{n}}} \frac{\partial v}{\partial t} \int_{0}^{1}(z u+(1-z) v)^{\frac{2-n}{n}} d z+\frac{2}{n} \frac{R_{\theta}}{u^{\frac{2}{n}}}
$$

are continuous functions. Since $u$ and $v$ are solutions of the CR Yamabe flow (4.2), it follows from the assumptions of Theorem 4.6 that $b$ is bounded from the above. Let $\bar{w}=e^{-\lambda t} w$. Then we have

$$
\begin{aligned}
\frac{\partial}{\partial t} \bar{w} & =e^{-\lambda t} \frac{\partial w}{\partial t}-\lambda e^{-\lambda t} w \\
& =e^{-\lambda t}\left(\frac{\partial w}{\partial t}-\lambda w\right) \\
& =e^{-\lambda t}\left[a \Delta_{\theta_{0}} w-(b+\lambda) w\right] \\
& =a \Delta_{\theta_{0}} \bar{w}-(b+\lambda) \bar{w} .
\end{aligned}
$$

Thus, we choose $\lambda>0$ sufficiently large such that $b+\lambda>0$. If $w$ is not identically zero on $M \times[0, T]$, then without loss of generality, we can assume $w>0$ somewhere, which means $\bar{w}>0$ at the same point. Since $\bar{w}(0) \equiv 0$, then by Theorem 4.5 we have $\bar{w} \leq 0$. This is a contradiction. Thus we obtain $\bar{w} \equiv 0$ on $M \times[0, T]$, which means $w \equiv 0$ on $M \times[0, T]$.

\section{The CR Yamabe Soliton}

By using Proposition 1.2, we can prove the following theorem. The corresponding theorem for the Yamabe soliton was proved in [13.

Theorem 5.1. Suppose $(M, \theta)$ is a 3 -dimensional noncompact $C R$ Yamabe soliton which is nonshrinking, i.e. $\mu \leq 0$. Assume that $\liminf _{x \rightarrow \infty} R_{\theta}(x) \geq 0$. Then the Tanaka-Webster scalar curvature $R_{\theta}$ of $(M, \theta)$ is nonnegative.

Proof. Suppose on the contrary that $\inf _{M} R_{\theta}<0$. Since $\liminf _{x \rightarrow \infty} R_{\theta}(x) \geq 0$ by assumption, there exists $x_{1} \in M$ such that

$$
R_{\theta}\left(x_{1}\right)=\inf _{M} R_{\theta}<0 .
$$

Then we have

$$
\Delta_{\theta} R_{\theta}\left(x_{1}\right) \geq 0, \nabla_{\theta} R_{\theta}\left(x_{1}\right)=0 \text { and }\left(R_{\theta}\right)_{0}\left(x_{1}\right)=0 .
$$

Combining (1.7) and (5.2), we obtain

$$
2 R_{\theta}\left(R_{\theta}-\mu\right) \leq 0 \text { at } x_{1} .
$$


On the other hand, it follows from (5.1) and the assumption that $\mu \leq 0$ that

$$
2 R_{\theta}\left(R_{\theta}-\mu\right)=2 R_{\theta}^{2}-2 \mu R_{\theta}>0 \text { at } x_{1},
$$

which contradicts to (5.3). This proves that $\inf _{M} R_{\theta} \geq 0$, as required.

Next, we consider the CR Yamabe soliton on the Heisenberg group $\mathbb{H}^{n}$ equipped with the standard contact form. The standard contact form $\theta_{0}$ on the Heisenberg group $\mathbb{H}^{n}$ is given by

$$
\theta_{0}=d t+\sqrt{-1} \sum_{i=1}^{n}\left(z_{i} d \bar{z}_{i}-\bar{z}_{i} d z_{i}\right)
$$

Note that both of the Tanaka-Webster scalar curvature and torsion of $\left(\mathbb{H}^{n}, \theta_{0}\right)$ vanish. Hence, it follows from (1.6) that any CR Yamabe soliton $\left(\mathbb{H}^{n}, \theta_{0}, f\right)$ is given by

$$
\frac{1}{2} f_{0}=\mu \text { and } f_{\alpha \alpha}=0 \text { for all } \alpha=1,2, \ldots, n,
$$

for some real-valued function $f$ and some constant $\mu$. The following theorem classifies all the CR Yamabe solition $\left(\mathbb{H}^{n}, \theta_{0}, f\right)$.

Theorem 5.2. Suppose that $\left(\mathbb{H}^{n}, \theta_{0}, f\right)$ is a CR Yamabe soliton. Then the potential function $f$ must be in the form of

$$
f(z, t)=2 \mu t+\sum_{p_{i}, q_{i}=0,1} C_{p_{1} q_{1} \cdots p_{n} q_{n}} z_{1}^{p_{1}} \bar{z}_{1}^{q_{1}} z_{2}^{p_{2}} \bar{z}_{2}^{q_{2}} \cdots z_{n}^{p_{n}} \bar{z}_{n}^{q_{n}}
$$

where $C_{p_{1} q_{1} \cdots p_{n} q_{n}}$ are constants such that $f$ is a real-valued function.

For example, we can take

$C_{p_{1} q_{1} \cdots p_{n} q_{n}}= \begin{cases}C, & \text { when } p_{i}=q_{i}=1 \text { and } p_{j}=q_{j}=0 \text { for } j \neq i, \text { where } 1 \leq i \leq n ; \\ 0, & \text { otherwise. }\end{cases}$

for some real constant $C$ to get $f(z, t)=2 \mu t+C|z|^{2}$ satisfying (5.4), which has already been pointed out in [1].

Proof of Theorem 5.2. To prove Theorem 5.2, we are going to show that any real function $f$ satisfying (5.4) must be in the form of (5.5). We integrate the first equation in (5.4) to get

$$
f(z, t)=2 \mu t+g
$$

for some real-valued function $g$ depending on $z$ and $\bar{z}$. Combining this with the second equation in (5.4), we get $g_{\alpha \alpha}=g_{\bar{\alpha} \bar{\alpha}}=0$ for all $\alpha=1,2, \ldots, n$. This implies

$$
g_{\alpha} \text { does not depend on } z_{\alpha} \text {, or equivalently, } g_{\bar{\alpha}} \text { does not depend on } \bar{z}_{\alpha} \text {. }
$$

Note that (see (2.14) in [1])

$$
g_{\alpha \beta}=g_{\beta \alpha} \text { and } g_{\alpha \bar{\beta}}-g_{\bar{\beta} \alpha}=\sqrt{-1} h_{\alpha \bar{\beta}} g_{0},
$$

where $h_{\alpha \bar{\beta}}$ is given by

$$
d \theta=\sqrt{-1} h_{\alpha \bar{\beta}} \theta_{\alpha} \wedge \theta_{\bar{\beta}} .
$$

Since $g$ does not depend on $t$, we have $g_{0}=0$. Hence, it follows from (5.8) that

$$
g_{\alpha \beta}=g_{\beta \alpha} \text { and } g_{\alpha \bar{\beta}}=g_{\bar{\beta} \alpha} \text { for all } \alpha, \beta .
$$

It follows from (5.7) that $g_{\beta}$ does not depend on $z_{\beta}$, which implies that $g_{\beta \alpha}$ does not depend on $z_{\beta}$ for all $\alpha$. Since $g_{\alpha \beta}=g_{\beta \alpha}$ by (5.9), we can conclude that $g_{\alpha \beta}$ 
does not depend on $z_{\beta}$ for all $\alpha$. Integrating $g_{\alpha \beta}$ with respect to $z_{\beta}$, we have, for any $\alpha=1,2, \ldots, n$,

$$
g_{\alpha}=E^{\beta}(z) z_{\beta}+F^{\beta}(z)
$$

for some functions $E^{\beta}$ and $F^{\beta}$ which do not depend on $z_{\beta}$. On the other hand, by (5.7), $g_{\bar{\beta}}$ does not depend on $\bar{z}_{\beta}$, which implies that $g_{\bar{\beta} \alpha}$ does not depend on $\bar{z}_{\beta}$. Since $g_{\alpha \bar{\beta}}=g_{\bar{\beta} \alpha}$ by (5.9), we can conclude that, $g_{\alpha \bar{\beta}}$ does not depend on $\bar{z}_{\beta}$ for all $\alpha$. Integrating $g_{\alpha \bar{\beta}}$ with respect to $z_{\beta}$, we have, for any $\alpha=1,2, \ldots, n$,

$$
g_{\alpha}=H^{\beta}(z) \bar{z}_{\beta}+I^{\beta}(z) .
$$

for some functions $H^{\beta}$ and $I^{\beta}$ which do not depend on $\bar{z}_{\beta}$. Differentiating (5.10) with respect to $z_{\alpha}$ and using the fact that $g_{\alpha \alpha}=0$, we obtain

$$
0=\frac{\partial E^{\beta}(z)}{\partial z_{\alpha}} z_{\beta}+\frac{\partial F^{\beta}(z)}{\partial z_{\alpha}}
$$

for $\beta \neq \alpha$. It follows from (5.12) that $\frac{\partial E^{\beta}(z)}{\partial z_{\alpha}}=\frac{\partial F^{\beta}(z)}{\partial z_{\alpha}}=0$ for $\beta \neq \alpha$. In particular, for any $\beta \neq \alpha, E^{\beta}(z)$ and $F^{\beta}(z)$ does not depend on $z_{\alpha}$. Hence, we can integrate (5.10) with respect to $z_{\alpha}$ to obtain

$$
g(z)=E^{\beta}(z) z_{\beta} z_{\alpha}+F^{\beta}(z) z_{\alpha}+G^{\beta}(z) \text { when } \beta \neq \alpha,
$$

for some function $G^{\beta}$ which does not depend on $z_{\alpha}$ and some functions $E^{\beta}$ and $F^{\beta}$ which do not depend on $z_{\beta}$ and $z_{\alpha}$. Similarly, differentiating (5.11) with respect to $z_{\alpha}$ and using the fact that $g_{\alpha \alpha}=0$, we obtain

$$
0=\frac{\partial H^{\beta}(z)}{\partial z_{\alpha}} \bar{z}_{\beta}+\frac{\partial I^{\beta}(z)}{\partial z_{\alpha}}
$$

for all $\beta$. It follows from (5.14) that $\frac{\partial H^{\beta}(z)}{\partial z_{\alpha}}=\frac{\partial I^{\beta}(z)}{\partial z_{\alpha}}=0$ for all $\beta$. In particular, for any $\beta, H^{\beta}(z)$ and $I^{\beta}(z)$ does not depend on $z_{\alpha}$. Hence, we can integrate (5.11) with respect to $z_{\alpha}$ to obtain

$$
g(z)=H^{\beta}(z) \bar{z}_{\beta} z_{\alpha}+I^{\beta}(z) z_{\alpha}+J^{\beta}(z)
$$

for some function $J^{\beta}$ which does not depend on $z_{\alpha}$ and some functions $H^{\beta}$ and $I^{\beta}$ which do not depend on $\bar{z}_{\beta}$ and $z_{\alpha}$. Now (5.5) follows from (5.13) and (5.15).

We have the following transformation law for the Tanaka-Webster scalar curvature and the torsion: if $\hat{\theta}=e^{2 u} \theta$, then we have (see Lemma 5.6 and Proposition 5.15 in [11)

$$
\begin{aligned}
e^{2 u} \widehat{R} & =R+2(n+1) \Delta_{\theta} u-4 n(n+1) u_{\alpha} u^{\alpha}, \\
\widehat{A}_{\alpha \beta} & =e^{-2 u}\left(A_{\alpha \beta}+2 i u_{\alpha \beta}-4 i u_{\alpha} u_{\beta}\right) .
\end{aligned}
$$

It follows from (5.16) that the Tanaka-Webster scalar curvature and torsion of $\left(\mathbb{H}^{n}, \hat{\theta}=e^{2 u} \theta_{0}\right)$ are given by

$$
\begin{aligned}
\widehat{R} & =e^{-2 u}\left(2(n+1) \Delta_{\theta_{0}} u-4 n(n+1) u_{\alpha} u^{\alpha}\right), \\
\widehat{A}_{\alpha \beta} & =e^{-2 u}\left(2 i u_{\alpha \beta}-4 i u_{\alpha} u_{\beta}\right) .
\end{aligned}
$$


Also, the vector field $\hat{T}$, the frame and the connection of $\hat{\theta}=e^{2 u} \theta_{0}$ are given by (see (5.7) and (5.14) in [11])

$$
\begin{aligned}
\hat{Z}_{\alpha}= & e^{-u} Z_{\alpha}, \hat{T}=e^{-2 u}\left(T+2 i u^{\bar{\gamma}} Z_{\bar{\gamma}}-2 i u^{\gamma} Z_{\gamma}\right) \text { and } \\
{\widehat{\omega_{\beta}}}^{\alpha}= & \omega_{\beta}{ }^{\alpha}+2\left(u_{\beta} \theta^{\alpha}-u^{\alpha} \theta_{\beta}\right)+\delta_{\beta}^{\alpha}\left(u_{\gamma} \theta^{\gamma}-u^{\gamma} \theta_{\gamma}\right) \\
& +i\left(u_{\beta}^{\alpha}+u_{\beta}{ }^{\alpha}+4 f_{\beta} f^{\alpha}+4 \delta_{\beta}^{\alpha} u_{\gamma} u^{\gamma}\right) \theta .
\end{aligned}
$$

It follows from (5.18) that

$$
\begin{aligned}
\hat{f}_{\alpha \alpha} & =\hat{Z}_{\alpha} \hat{Z}_{\alpha} f-{\widehat{\omega_{\beta}}}^{\alpha}\left(\hat{Z}_{\alpha}\right) f \\
& =e^{-u} Z_{\alpha}\left(e^{-u} Z_{\alpha} f\right)-\omega_{\alpha}^{\alpha}\left(e^{-u} Z_{\alpha}\right)\left(e^{-u} Z_{\alpha} f\right)-3 u_{\alpha} \theta^{\alpha}\left(e^{-u} Z_{\alpha}\right)\left(e^{-u} Z_{\alpha} f\right) \\
& =e^{-2 u} f_{\alpha \alpha}+e^{-u} Z_{\alpha}\left(e^{-u}\right) Z_{\alpha} f-3 e^{-2 u} u_{\alpha} f_{\alpha} \\
& =e^{-2 u}\left(f_{\alpha \alpha}-4 u_{\alpha} f_{\alpha}\right) .
\end{aligned}
$$

Now suppose that $\left(\mathbb{H}^{n}, \hat{\theta}=e^{2 u} \theta_{0}, f\right)$ is a CR Yamabe soliton. Then, by (1.6), (5.17)-(5.19), we have

$$
\begin{aligned}
& e^{-2 u}\left(2(n+1) \Delta_{\theta_{0}} u-4 n(n+1) u_{\alpha} u^{\alpha}\right)+\frac{1}{2} e^{-2 u}\left(f_{0}+2 i u^{\bar{\gamma}} f_{\bar{\gamma}}-2 i u^{\gamma} f_{\gamma}\right)=\mu, \\
& e^{-2 u}\left(f_{\alpha \alpha}-4 u_{\alpha} f_{\alpha}\right)-e^{-2 u}\left(2 u_{\alpha \alpha}-4 u_{\alpha}^{2}\right) f=0 \text { for all } \alpha=1,2, \ldots, n .
\end{aligned}
$$

If $u$ depends only on $t$, then (5.20) reduces to

$$
\frac{1}{2} e^{-2 u} f_{0}=\mu \text { and } f_{\alpha \alpha}=0 \text { for all } \alpha=1,2, \ldots, n .
$$

Integrating the first equation in (5.21) with respect to $t$, we obtain

$$
f(z, t)=2 \mu \int e^{2 u} d t+g
$$

for some real-valued function $g$ depending only on $z$ and $\bar{z}$. Submitting this into the second equation of (5.21), we get $g_{\alpha \alpha}=g_{\bar{\alpha} \bar{\alpha}}=0$ for all $\alpha=1,2, \ldots, n$. Now, we can follow the proof of Theorem 5.2 to conclude the following:

Corollary 5.3. Suppose that $\left(\mathbb{H}^{n}, e^{2 u} \theta_{0}, f\right)$ is a CR Yamabe soliton such that $u$ is a function depending only on $t$. Then the potential function $f$ must be in the form of

$$
f(z, t)=2 \mu \int e^{2 u} d t+\sum_{p_{i}, q_{i}=0,1} C_{p_{1} q_{1} \cdots p_{n} q_{n}} z_{1}^{p_{1}} \bar{z}_{1}^{q_{1}} z_{2}^{p_{2}} \bar{z}_{2}^{q_{2}} \cdots z_{n}^{p_{n}} \bar{z}_{n}^{q_{n}}
$$

where $C_{p_{1} q_{1} \cdots p_{n} q_{n}}$ are constants such that $f$ is a real-valued function.

\section{REFERENCES}

1. H. D. Cao, S. C. Chang and C. W. Chen, On three-dimensional CR Yamabe solitons. J. Geom. Anal. 28 (2018), 335-359.

2. H. D. Cao, S. C. Chang and C. W. Chen, $C_{0}$-positivity and a classification of closed threedimensional CR torsion solitons. preprint. https://arxiv.org/abs/1902.11264

3. S. C. Chang, H. L. Chiu, and C. T. Wu, The Li-Yau-Hamilton inequality for Yamabe flow on a closed CR 3-manifold. Trans. Amer. Math. Soc. 362 (2010), 1681-1698.

4. P. T. Ho, A note on compact CR Yamabe soliton. J. Geom. Phys. 94 (2015), 32-34.

5. P. T. Ho, Result related to prescribing pseudo-Hermitian scalar curvature. Int. J. Math. 24 (2013), 29pp. 
6. P. T. Ho, The long time existence and convergence of the CR Yamabe flow. Commun. Contemp. Math. 14 (2012), 50 pp.

7. P. T. Ho, The Webster scalar curvature flow on CR sphere. Part I. Adv. Math. 268 (2015), 758-835.

8. P. T. Ho and S. Kim, The Yamabe problem on noncompact CR manifolds. Pacific J. Math. 285 (2016), 375-391.

9. P. T. Ho, W. Sheng and K. Wang, Convergence of the CR Yamabe flow. Math. Ann. 373 (2019), 743-830.

10. D. Jerison and J. M. Lee, The Yamabe problem on CR manifolds. J. Differential Geom. 25 (1987), 167-197.

11. J. M. Lee, The Fefferman metric and pseudo-Hermitian invariants. Trans. Amer. Math. Soc. 296 (1986), 411-429.

12. L. Ma and Y. An, The maximum principle and the yamabe flow. Partial Differential Equations and Their Applications (1999), 211-224.

13. L. Ma and V. Miquel, Remarks on scalar curvature of Yamabe solitons. Ann. Global Anal. Geom. 42 (2012), 195-205.

14. W. Sheng and K. Wang, The exponential convergence of the CR Yamabe flow. Sci China Math 63 (2020), 979-992.

15. N. Tanaka, A differential geometric study on strongly pseudo-convex manifolds, Kinokuniya, (1975) Tokyo.

16. S. M. Webster, Pseudohermitian structures on a real hypersurface, J. Differential Geom. 13 (1978), 25-41.

Department of Mathematics, Sogang University, Seoul 04107, Korea

E-mail address: ptho@sogang.ac.kr, paktungho@yahoo.com.hk

College of Sciences, China Jiliang University, Hangzhou 310018, China.

E-mail address: wangkb@cjlu.edu.cn, $21235005 @ z j u . e d u . c n$ 